\title{
Métropoles maghrébines, dossier coordonné par Alison Rice
}

\section{Claudia Mansueto}

\section{(2) OpenEdition \\ 1 Journals}

\section{Edizione digitale}

URL: http://journals.openedition.org/studifrancesi/5793

DOI: 10.4000/studifrancesi.5793

ISSN: 2421-5856

Editore

Rosenberg \& Sellier

\section{Edizione cartacea}

Data di pubblicazione: 1 septembre 2011

Paginazione: 466-467

ISSN: 0039-2944

\section{Notizia bibliografica digitale}

Claudia Mansueto, «Métropoles maghrébines, dossier coordonné par Alison Rice», Studi Francesi [Online], 164 (LV | II) | 2011, online dal 30 novembre 2015, consultato il 08 janvier 2021. URL: http:// journals.openedition.org/studifrancesi/5793 ; DOI: https://doi.org/10.4000/studifrancesi.5793

Questo documento è stato generato automaticamente il 8 janvier 2021.

\section{(c) (i) (9)}

Studi Francesi è distribuita con Licenza Creative Commons Attribuzione - Non commerciale - Non opere derivate 4.0 Internazionale. 


\title{
Métropoles maghrébines, dossier coordonné par Alison Rice
}

\author{
Claudia Mansueto
}

\section{NOTIZIA}

Métropoles maghrébines, dossier coordonné par Alison RICE, «Expressions maghrébines», vol. 8, n. 2, hiver 2009, pp. 1-199.

1 Questo numero di «Expressions maghrébines» studia i rapporti sempre difficili che si intessono tra Francesi e Maghrebini all'interno delle principali città dell'Esagono. Alison RICE, autrice dell'introduzione (pp.1-7), ricorda, infatti, che «les villes ont toujours été les lieux privilégiés des rencontres franco-maghrébines» (p.1); spazi urbani vissuti da chi li occupa sia come barriere invalicabili che come «espaces ouverts et expansifs» (p. 5). È proprio questa percezione ambivalente dello spazio cittadino ad interessare intellettuali, cantanti e scrittori concordi nel sostenere la neutralità aprioristica della città, foglio bianco che viene "scritto" ogni giorno dai suoi abitanti.

Il numero comprende due sezioni. La prima, «Articles» (pp.11-150) fa conoscere al lettore il caleidoscopio cittadino in cui si riflettono le speranze di chi vive ai margini, in una banlieue sempre più sinonimo di un indifferente e, per certi versi, rassicurante ailleurs; la seconda, «Varia» (pp.155-189), riunisce tre articoli scritti in occasione dell'esposizione di Lione dell'agosto 2009, Générations: un siècle d'histoire culturelle des Maghrébins de France.

3 La prima sezione è composta da otto saggi: Le Paris arabe des «Boucs», des «Laskars» et des «Chibanis» (Chraibi, Boudjedra et Djemaï) di Florian ALIX (pp. 11-30); C'est plus la médina que Zola: writing Maghrebian Paris through la Goutte-d'Or di Lisa wEIss (pp. 31-48); Habel à la merci de Paris: une lecture d'Habel de Mohammed Dib di Laurence DENIÉ-HIGNEY (pp. 49-65); Paris dans l'ouvre de Tahar Ben Jelloun di Bernard URBANI (pp. 67-80); Impenetrable Paris: Leila Marouane's La Vie sexuelle d'un islamiste à Paris di Alison RICE (pp. 81-97); South of the Capital: Geographies of Resistance in Maghrebi-french film di Claudia ESPOSITO (pp. 99-109); 
Rock el'Casbah!: identité et espace urbain chez Rachid Taha di Christa JONES (pp. 111-129) e «Toulouse Sarrazine» di Danielle MARX-SCOURAS (pp. 131-150).

Florian Alix, analizzando i romanzi Les boucs di Chraïbi (1989), Topographie idéale pour une agression caractérisée di Boudjedra (1986) e Gare du Nord di Djemaï (2006), rileva (ma è cosa nota) che Parigi è diventata progressivamente un vero e proprio centro di immigrazione, un polo di riferimento per migranti maghrebini che vedono nella fredda «Paris-méduse» (p.4) un nuovo Eldorado. Come Voltaire e Montesquieu già sostenevano nel secolo dei Lumi, Alix sembra essere convinto che, per capire una realtà, bisogna ascoltare chi entra in quella dimensione per la prima volta con gli occhi ingenui di chi ignora tutto dell'ambiente che lo accoglie. Lasciando la parola agli ignari protagonisti dei tre romanzi, lo studioso aiuta il lettore a vedere il vero volto, o almeno un volto inedito, della sfavillante Ville-Lumière: Parigi non accoglie gli immigrati, ma li ammassa in quartieri-ghetto che rappresentano la negazione di tutti quei sogni di riscatto nutriti per tanti anni. Relegati nei margini, bloccati nei sotterranei della città, dimenticati ed ignorati dai parigini indifferenti, limitati da divieti incomprensibili, gli immigrati restano imprigionati come tanti goffi elefanti in una cristalliera. Se è vero, come ricordava Alison Rice nell'introduzione, che la città è un foglio bianco da riempire con le parole di chi vi abita, Parigi appare come «un espace lettré illisible» (p. 21) per i maghrebini. Alix, infatti, evidenzia come gli immigrati si muovano costantemente in un buio conoscitivo irreversibile: circondato da parole sconosciute, «le migrant nu» (p. 22) non ha speranza di accedere al mondo civilizzato, di entrare in quella Parigi vorticosa dove non c'è spazio per poveri e lenti invertebrati analfabeti.

Nel secondo articolo, Lisa Weiss si sofferma sul rapporto che si stabilisce tra città e memoria. Accantonati negli storici quartieri di Belleville e de La Goutte-d'Or, gli immigrati tendono a riprodurre nei nuovi spazi i ritmi e le abitudini acquisite durante l'infanzia nei propri paesi d'origine. Queste propaggini arabe ridisegnano così l'identità di Parigi e ciò che era unitario e definito sta diventando multiforme ed ibrido. In quanto prolungamenti artificiali delle grandi città maghrebine, i quartieri arabi di Parigi hanno conosciuto la violenza degli harkis, le spie algerine a tempi della guerra di indipendenza, l'entusiasmo dei militanti per la libertà e il dolore per chi è rimasto ucciso dall'indifferenza di una Francia sorda alle grida provenienti dai sobborghi. «C'était la guerre. Ici. En plein Paris... une guerre ouverte et en même temps personne n'en parlait» (p. 41) ricorda uno dei personaggi de Les Caves de la Goutte-d'Or di Gérard Streiff (2001) per ribadire le atrocità di una guerra dimenticata, di uno scempio che si consumava nel cuore delle Francia senza che nessuno se ne accorgesse. A conclusione del suo articolo, Lisa Weiss sostiene che la seconda generazione di immigrati prova un certo affetto per quelle strade e quei quartieri in cui il dolore e lo smarrimento degli antenati sono rimasti impressi come inchiostro indelebile su un foglio tristemente bianco. Lieux de la mémoire privilegiati, i quartieri dell'immigrazione raccontano le storie degli invisibili di ieri e dei disorientati di oggi, e offrono «a deeper portrait of the identity of Paris» (p. 47). In Habel à la merci de Paris: une lecture d'Habel de Mohammed Dib, Laurence Denié-Higney inserisce un altro tassello per completare il disegno della nuova Parigi-mosaico. Lo studioso fa notare che per Mohammed Dib - l'autore di cui si occupa nel suo articolo - « la ville est une pratique personnelle» (p. 51), uno spazio che assume connotazioni assolutamente soggettive, a seconda dei «chemins imprévus» (p. 51) che sceglie di percorrere. Non più paralizzato, il migrante si muove alla scoperta della città, metafora della propria identità. Denié-Higney stabilisce proprio questo parallelo tra 
spazi urbani e mondo interiore: esplorando ciò che è ignoto, abbracciando l'ideale del casuale che disorienta i progetti personali, l'uomo è più predisposto ad accettare quel lato oscuro della propria personalità che, sfuggendo ad ogni logica, destabilizza. In conclusione, Denié-Higney ricorda la positività dell'errance: è solo perdendo la voie che si ritrova la propria voix interiore.

6 L'articolo di Bernard Urbani, Paris dans l'œuvre de Tahar Ben Jelloun, insiste sull'estrema solitudine dell'immigrato che arriva per la prima volta a Parigi. «Mêlé[e]s à toute une poliphonie de voix» (p. 73), i maghrebini, che vivono nei sordidi sobborghi parigini, si riuniscono per rievocare le bellezze dei luoghi dell'infanzia ormai lontani, per aiutarsi ad accettare il nuovo destino segnato dalla negazione di ogni felicità, anche futura. Per Tahar Ben Jelloun, come sottolinea Urbani, Parigi è una città tragica, quasi maledetta, una sorta di vampiro che succhia all'uomo ogni aspettativa ed ogni desiderio. Il modo migliore per rappresentare Parigi, regno dell'«aliénation totale» (p. 74), sarebbe pertanto quello di scegliere una scrittura illeggibile, capace, nella sua allucinante schizofrenia, di tradurre al meglio l'infernale mostruosità della capitale francese. Il ritratto negativo della Parigi di Ben Jelloun nell'interpretazione di Urbani, diventa ancora più crudo nell'articolo di Alison Rice Impenetrable Paris: Leila Marouane's La Vie sexuelle d'un islamiste à Paris, che riflette sul dramma vissuto da tutti coloro che, come il protagonista del romanzo di Leilla Marouane, credono che basti cambiare quartiere perché cessino i pregiudizi razziali che condizionano l'esistenza di un immigrato. Il protagonista del romanzo, Mohamed, desideroso di conoscere il cuore pulsante della capitale francese, decide di uscire dai sobborghi in cui viveva per avventurarsi nel più elegante sesto arrondissement, ma Parigi non integra, rifiuta il diverso e soprattutto non tollera che ci si possa fabbricare un altro viso, come sosteneva Ofelia nell'Amleto.

7 Parigi è dunque un bottino inaccessibile per gli immigrati maghrebini, mentre città come Marsiglia e Tolosa, nascenti capitali del Sud, appaiano come i nuovi regni del multiculturalismo, di quell'agognato melting pot irrealizzabile altrove. I già citati articoli di Claudia Esposito, di Christa Jones e di Danielle Marx-Scouras mostrano come registi e musicisti siano orientati ad esplorare nuove megalopoli in cui gli spazi siano finalmente per loro agevoli ponti di comunicazione orizzontale e non insormontabili barricate verticali. Claudia Esposito presentando i film Bye-Bye di Karim Dridi (1995) e La graine et le mulet di Abdellatif Kechiche (2007) insiste sull'importanza di vivere la multiculturalità come uno stimolo costante perché gli spazi cittadini «are not neutral, they are continually visited, revisited, coded and re-coded» (p. 108). La musica è forse l'unico linguaggio che può favorire l'espansione di una territorialità più ibrida, meno legata a radici ideologiche: la mescolanza dei generi musicali privilegiati da Rachid Taha, presentato da Christa Jones o lo straordinario fermento culturale di Tolosa descritto da Danielle Marx-Scouras, sono esempi tangibili di come un'alternativa sia possibile, un'apertura al multiplo e al plurale non sia solo utopica retorica: «Nous sommes donc tous invités à développer une curiosité pour "l'autre". Lorsque nous rencontrons des gens d'autres cultures, nous sommes tous invités à réfléchir à notre propre situation d'immigrés permanents et à nouer des contacts avec «l'autre». De cette rencontre, si elle est fructueuse, peut naître un esprit de solidarité. C'est en traversant les frontières que l'artiste et par extension, nous, ses fans, pouvons traverser les frontières érigées à l'intérieur de nous-mêmes» (p. 127). 